\title{
Unkomplizierte Zystitiden: Senföle einsetzen und Antibiotikaresistenzen vermeiden
}

— Die bei akuten Zystitiden am häufigsten verordneten Antibiotika sind Folsäure-Antagonisten wie Cotrimoxazol oder Fluorchinolone wie Ciprofloxacin. Die Resistenzsituation bei diesen Antibiotika hat sich in den letzten Jahren zunehmend verschlechtert, nicht nur unter Uropathogenen, wie den gramnegativen E. coli. Vor allem die steigenden MRSARaten im Zusammenhang mit der Verschreibung von Fluorchinolonen sind besorgniserregend. Daher wird empfohlen, diese hoch wirksamen Substanzen für ernstere Erkrankungen zurückzuhalten. Dementsprechend hat die Deutsche Gesellschaft für Urologie in den 2010 verabschiedeten S3-Leitlinien empfohlen, Cephalosporine und Fluorchinolone bei der unkomplizierten Zystitis nicht als Antibiotika der ersten Wahl einzusetzen, solange es therapeutische Alternativen mit vergleichbarer Effizienz und akzeptablem Nebenwirkungsspektrum gibt.

Vor diesem Hintergrund wurde in einer aktuellen JAMA-Studie [Hooton TM et al. JAMA 2012 307(6): 583-9] untersucht, ob man bei unkomplizierten Harnwegsinfekten auf das ß-Laktam-Antibiotikum Cefpodoxim zurück- greifen kann - eine unter dem Gesichtspunkt der Resistenzproblematik besser vertretbare Alternative als Ciprofloxacin. Das enttäuschende Ergebnis: In der randomisierten, doppelblinden Studie zeigte sich Cefpodoxim bei einer Kurzzeittherapie über drei Tage dem Fluorchinolon deutlich unterlegen.

„In der Natur gibt es aber noch Stoffe, die auch bei Problemkeimen wirksam sind, so zum Beispiel die Senföle aus Kapuzinerkresse und Meerrettich", erklärte Prof. Uwe Frank, Heidelberg. Wie Studien belegen, zeigen die Senföle aus Kapuzinerkressenkraut und Meerrettichwurzel (ANGOCIN ${ }^{\circledR}$ Anti-Infekt N) bei akuten Harnwegsinfekten eine hohe Wirksamkeit, bei einem gleichzeitig hohen Verträglichkeits- und Sicherheitsprofil [u.a. Albrecht, U. et al. 2007; Curr Med Res Opin 23(10): 2415-22]. Wie die Fluorchinolone haben auch die Senföle ein breites antibakterielles Wirkspektrum im grampositiven und gramnegativen Bereich, wie verschiedene In-vitro-Studien am Universitätsklinikum Freiburg dokumentieren [u.a. Conrad $A$ et al. Z Phytotherapie 2008; 29: Suppl.1: S22-3]

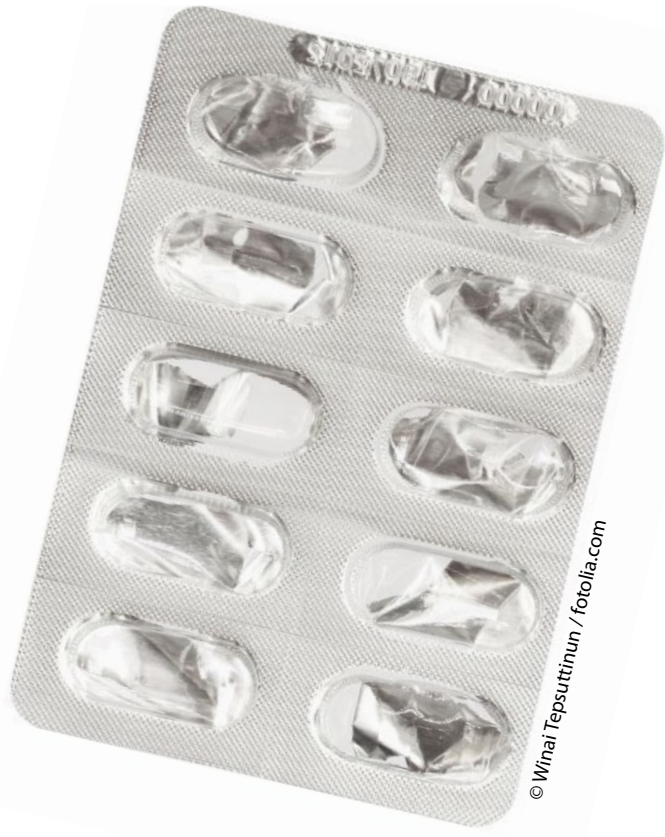

Der häufig unkritische Einsatz von Antibiotika ist problematisch. Oft gibt es Alternativen wie Pflanzenpräparate.

Sogar gegen resistente Formen von E.coli und Problemkeime wie MRSA war das Senfölgemisch wirksam.

Nach Informationen von

Repha, Langehagen

\section{Erektile Dysfunktion:}

\section{PDE-5-Hemmer auch für Patienten mit stabiler KHK geeignet}

— Gibt es für Patienten mit einer erektilen Dysfunktion (ED) eine Rückkehr zu einer fast natürlichen Sexualität? Diese Frage war wesentlicher Teil des Lilly.Satellitensymposiums beim diesjährigen Kongress der European Association of Urology (EAU) in Paris. Die Referenten betonten dabei, dass die Konstanztherapie mit Tadalafil $5 \mathrm{mg}$ den Betroffenen ein hohes Maß an Flexibilität und somit ein Sexualleben fast wie früher ermöglichen kann. Aktuell vorgestellte Daten unterstreichen, dass sich die Therapie mit Tadalafil (Cialis ${ }^{\oplus}$ ) positiv auf das Sexualleben der Betroffenen auswirkt [Rubio-Aurioles $\mathrm{E}$ at al. J Sex Med 2012; Epub ahead of print].

Die EAU-Leitlinien beschreiben PDE-5-Hemmer als First-Line-Therapie für ED-Patienten [Hatzimouratidis K Eur Urol 2010; 57: 80414]. Tadalafil (Cialis ${ }^{\oplus}$ ) ermöglicht dabei als einziges Präparat der Wirkstoffklasse eine
Konstanztherapie: Bei einer täglichen Einnahme des Präparats in der niedrigen 5 mgDosierung baut sich - Ansprechen auf die Therapie vorausgesetzt - in der Regel innerhalb von fünf Tagen ein konstanter Wirkstoffspiegel auf (Halbwertszeit des Wirkstoffs: 17,5 Stunden). Die sexuelle Aktivität ist dadurch zeitlich nicht mehr unmittelbar an die Einnahme der Tablette gekoppelt. Daher eigne sich diese Behandlungsoption vor allem für Paare, die sich ein möglichst spontanes Sexualleben wünschen, so die Autoren der EAU-Leitlinien.

Die Referenten des Satellitensymposiums thematisierten neben der Wirksamkeit der ED-Therapie mit Tadalafil auch die Verträglichkeit dieser Behandlungsoption bei Patienten mit Begleiterkrankungen. Der Aspekt der Komorbidität sei von besonderer Bedeutung, da die ED häufig mit Diabetes, einem benignen Prostatasyndrom (BPS) oder mit kardiovaskulären Erkrankungen einhergehe [Braun $\mathrm{M}$ et al. Int J Impoz Res 2000; 12: 305-11]. Zurückzuführen ist das gemeinsame Auftreten der ED mit den genannten Begleiterkrankungen auf ähnliche Risikofaktoren. Die American Heart Association (AHA) empfiehlt in einer aktuellen Publikation, auch Patienten, die neben einer ED eine stabile KHK aufweisen, mit PDE 5-Hemmern zu behandeln [Levine GN et al. Circulation 2012; 125: 1058-1107]. In diesem Zusammenhang betont die AHA die Bedeutung der Sexualität für die generelle Lebensqualität der betroffenen Patienten und ihrer Partnerinnen.

Nach Informationen von

Lilly, Bad Homburg 